\title{
Optimization of novel phase separating IBS process
}

\author{
Marco Jupé, ${ }^{\mathrm{a}, \mathrm{b}}$, Thomas Willemsen ${ }^{\mathrm{a}, \mathrm{b}}$, Sina Malobabic ${ }^{\mathrm{a}}$, Kornelia Schuba ${ }^{\mathrm{a}}$, Detlev Ristau ${ }^{\mathrm{a}, \mathrm{b}}$ \\ ${ }^{a}$ Laser Zentrum Hannover e.V., Hollerithallee 8, 30419 Hanover, Germany; \\ ${ }^{\mathrm{b}}$ QUEST: Centre of Quantum Engineering and Space-Time Research, Leibniz Universität Hannover, \\ Germany;
}

\begin{abstract}
During the last years, optical low loss components gained more and more industrial interest and led to novel approaches for the production in optical coating technology. The application of filtered deposition technologies promises a significant reduction of particle contamination. Usually, filtered techniques are applied in combination with processes which produce a high level of undesired particles, like the cathodic arc deposition. In the present contribution, a magnetic field filter is applied in combination with a high quality ion beam sputtering process. The focus of the investigation constitutes the modulation of the guiding process with respect to the guiding efficiency. Numerical investigations reveal the trajectories of the ions during the guiding process and allow to analyze the influence of the magnetic field and the resulting electrostatic potential. In this study, the guiding effect is observed to be dominated by the electric potential compared to the magnetic field. However according to the simulations, very high ion guiding efficiencies can be achieved using moderate magnetic fields and electrical potentials. Furthermore, it is demonstrated that the experimental and calculated efficiencies agree well. Consequently, the simulation is the basis for a further optimization of the filtered ion beam sputtering processes.
\end{abstract}

Keywords: Modeling, thin film growth, Lagrange formalism, Magnetic field separation, Filtered Ion Beam Sputtering

\section{INTRODUCTION}

Currently, thin film coating technology is applied in a wide range of research and industrial areas. Consequently, the specific demands on the coatings are varying in a broad spectrum. In particular, the requirements on optical components characterized by minimized particle content have experienced a significant increase in the last years. Especially in the manufacturing of laser optics, the reduction of particle contamination gains dramatically of importance, because $\mu \mathrm{m}$ and nanometer size defects are often responsible for laser induced damage in the thermal damage regime [1]. Furthermore, the defects may cause increased scattering and absorption which can induce ghost images and phase front dislocations. Consequently, the established coating procedures are optimized in respect to a significant reduction of the particle contamination. In the last decades, sputtering techniques have been proven to achieve highest quality coatings for high power and highest finesse applications like for example in the gravitational wave detection or optical clocks [2, 3]. Especially, Ion Beam Sputtering (IBS) was developed to a coating process with superior quality. Actually, absorption losses below $1 \mathrm{ppm}$ can be achieved and film thickness precisions of a few or even one atomic layer are realized. Nevertheless, new photonic technologies impose continuously increasing demands on optical components challenging the established processes including even advanced IBS technologies. The present state in achievable particle densities cannot meet the requirements of the next generation of optical devices and high power laser systems. The particles are usually generated in different phases of the manufacturing process of an optical component. Consequently, a precise analysis of each production step and its dedicated optimization is required. Especially for complex optical components with a high number of layers and a huge physical thickness, the coating process itself often contributes the major sources for particle contamination. This may apply especially to ion sputtering processes, where the debris produced during sputtering on the target surface is suggested to find its way to the growing layer. Taking the current state of the art into account, the present contribution considers a novel coating process, which specially separates the sputtering process and the thin film growth on the sample surface. In detail, the process applies magnetic field plasma guiding to transport the coating material from the target to the substrates. The set-up includes a curved separator which masks the direct line of sight between the target and the substrates. Generally, only ions of the deposition material components are guided by the magnetic field to the substrate. In contrast to this, heavy debris particles follow a linear ballistic trajectory and are not able to reach the substrates via the curved coil.

Optical Systems Design 2015: Advances in Optical Thin Films V, edited by Michel Lequime,

H. Angus Macleod, Detlev Ristau, Proc. of SPIE Vol. 9627, 96270K · @ 2015 SPIE

CCC code: 0277-786X/15/\$18 - doi: 10.1117/12.2191229

Proc. of SPIE Vol. 9627 96270K-1 
In the present contribution both, fundamental studies and experiments close to applications are presented. A theoretical model is presented to describe and gain the fundamental knowledge of the dynamics of the guiding process. Furthermore, the influence of the guiding on the deposition rate is investigated and effects of the axial magnetic as well as the electrostatic potential are analyzed. In conclusion, the separation of coating material can be considered as a promising novel approach for a significant reduction of the particle contamination in IBS-coatings with advantageous effects on their optical losses and the power handling capabilities.

\section{ION BEAM SPUTTERING AND GUIDING FACILITY}

The basic concept of the particle separation after sputtering had been investigated using different original deposition procedures. The first experiments were presented by Aksenov [3,4]. An axial magnetic field was used to guide a highly ionized plasma cathodic arc plasma from the arcing process to the substrates. In these experiments a significant reduction of the particle contamination was proven. Nowadays, filtered cathodic arc deposition techniques have been established especially for mechanical hard coatings and in some application in microelectronics [5]. It has to be mentioned that plasma arcing produce a significant amount of macro particles. Within the development of phase separating processes, various materials and different separator geometries are developed and optimized [6,7]. With respect to demands of high end dielectric optics, the initial material generation should create a possibly lower fraction of particles. In 2004, the phase separating process was combined with HIPIMS (High Power Impulse Magnetron Sputtering) [10]. Generally in comparison to the vacuum cathodic arc deposition process (VAD), the HIPIMS leads to a significantly reduced particle densities. Nevertheless for the manufacturing of highest quality components HIPIMS creates a considerable content of particles, which limits the application of the process. In contrast to the VAD and HIPIMS, the material in IBS is nearly neutral. Depending on the target material, the degree of ionization of the coating material is assumed to be in the low percent range. Nevertheless, the degree of ionization is increased by post sputtering ionization processes like impact ionization or coherent charge transfer and IBS has proven to manufacture optical coatings of the highest quality. Because of the geometric separation between ion source and target the influence of the magnetic field of the guiding coil and the RF-field of the ion source are not interacting, directly, which is helpful for a stable deposition process. Consequently, if a higher degree of ionization can be produced, the IBS-process delivers an extraordinary basis for a fundamental investigation of the guiding process and of the layer nucleation on the way towards a contamination free deposition process. Since 2008, a filtered ion beam sputtering (FIBS) process is developed and characterized. The schematically drawing of the coating process is displayed in fig. 1. The generation of the coating material flux and the deposition process are located in separate vacuum chambers which can be operated individually.

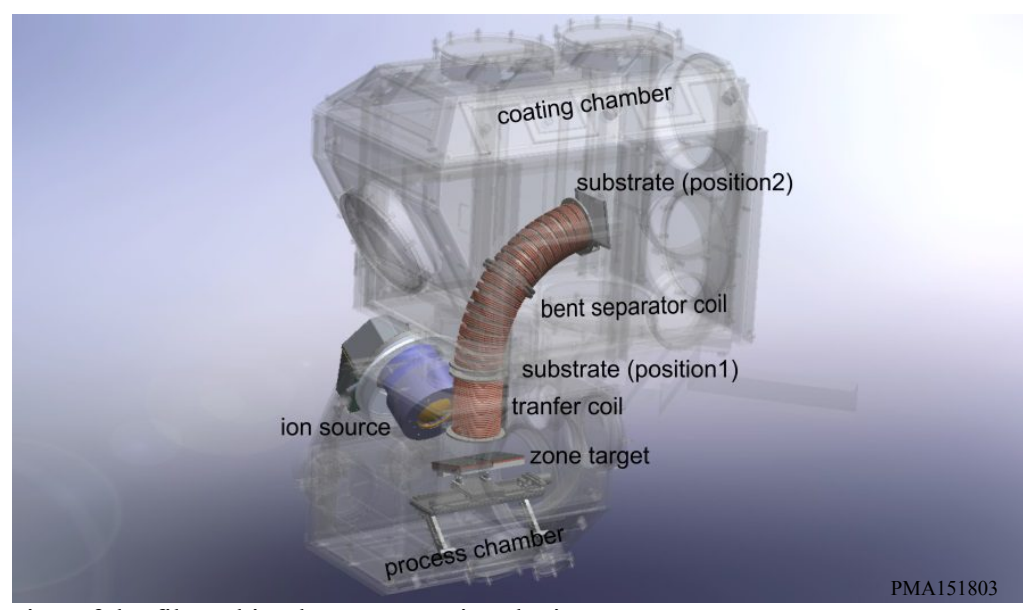

Figure 1: Schematical drawing of the filtered ion beam sputtering device.

The guiding system itself is divided in two magnetic field separator coils. The linear transfer coil is responsible to guide the material from the target to the exit of the process chamber. The separation coil masks the direct line of sight between the target and the sample. Consequently only guided material can be deposited on the substrate following a trajectory defined the magnetic field lines. Both coils have the same aperture of $18 \mathrm{~cm}$. The setup has two defined positions for plasma and deposition experiments, which are marked in fig. 1. The first position is located between transport and separation coil. This position is dedicated to fundamental investigation with respect to the guiding dynamics and 
efficiency. In this configuration the line of sight is not masked and the background of the non-guided material can be measured. The second position is addressed to investigate the reduction of particle contamination. In this configuration the line of sight is masked and the coating material flux is completely blocked without magnetic field. [11]. Both coils are able to create an axial magnetic field between 0 and $60 \mathrm{mT}$, individually. The basic IBS process is driven by a commercial ion source ("16 cm High Power" source from Veeco), and the beam is focused by an oval three grid ion extraction system which is optimized in respect to the entrance aperture of the magnetic guiding system. Ternary mixtures can be also manipulated by magnetic fields. These mixtures are generated by cosputtering of two water cooled metallic targets which are combined to a zone target on the target mount. In general up to four different materials can be applied on the mounting table, but usually only two or three materials are exposed to the ion beam in order to suppress parasitic sputtering effects.

\section{THEORETICAL DESCRIPTION OF THE GUIDING PROCESS}

Due to the physical nature of the process, only charged material particles can be guided. Both, the electrons and the ions are part of the process and have to be included in the calculation of the guiding process. In general, a spatial separation of negative charged electrons and the positively charged heavy ions or molecules is generated by the trapping of the electrons by the axial magnetic field. This spatial separation can be attributed to the Lorentz force compensating the centrifugal force. The electrons are constrained onto a spiral trajectory [12]. The diameter of the trajectory of the electrons is defined by the Larmor radius $\mathrm{r}_{\text {Larmor }}$ with a typical value of a few $\mathrm{mm}$. The Larmor radius for a single charged particle (mass $\mathrm{m}$; rotation velocity $v_{\perp}$ and elementary charge e) is given by:

$$
r_{\text {Larmor }}=m \cdot v_{\perp} /(\mathrm{e} \cdot|\vec{B}|)
$$

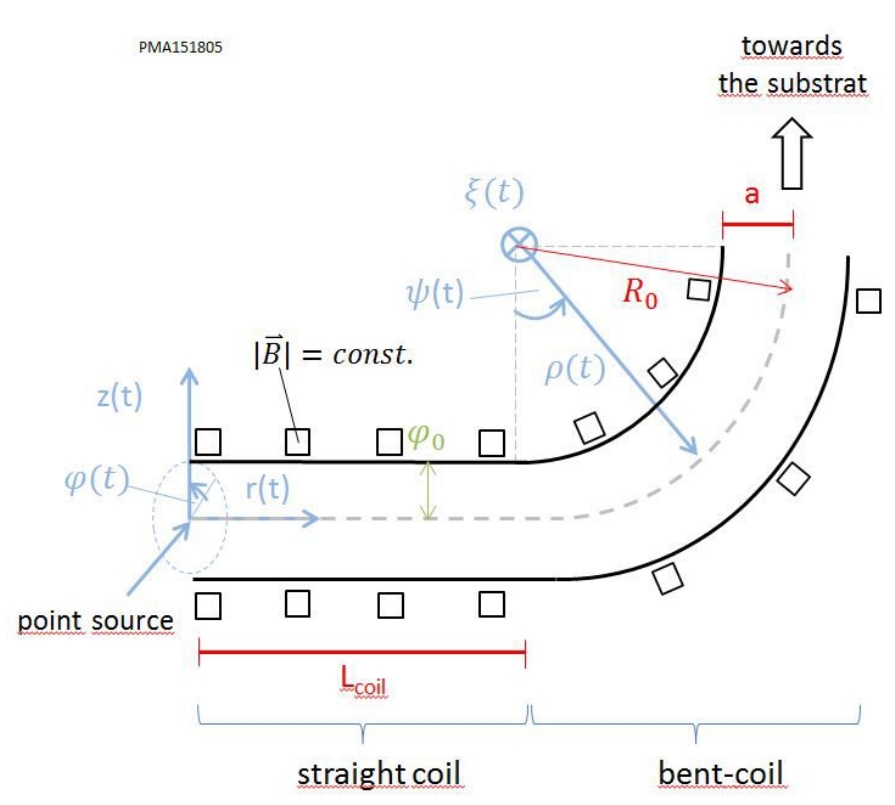

Figure 2: Coordinates of the guiding system
The diameter must be smaller than the dimension of the magnetic field coils to satisfy the guiding conditions. In this case the electrons are magnetized. In contrast to the electrons, the mass of the ions are too high and the trajectories of the ions are influenced marginally by the magnetic field $|\vec{B}|$, because the Larmor radius is larger than the aperture of the coils. However, because of the spatial separation of positive and negative charged particles, an electrostatic potential $\phi_{0}$ between the wall and the electron pipe is generated. In conjunction with the magnetic field the ions are forced to complex trajectories in chains along the field lines. For the theoretical description of the behavior, Aksenov developed a model based on the Lagrange formalism [3, 4]. This model was further developed and modified, which is the basis for the present contribution [13]. The solution of the complete problem requires a subdivision into two parts for the linear and the bent coil, respectively. The Lagrange equation $\mathrm{L}(\mathrm{r}(\mathrm{t}), \varphi(\mathrm{t}), \mathrm{z}(\mathrm{t}))$ for the linear part is given by cylindrical coordinates with radius $r$, a polar angle $\phi$ and a propagation in $\mathrm{z}$ direction by; 


$$
\begin{array}{rr}
\left.L=\left[\dot{r}(t)^{2}+(r(t) \cdot \dot{\varphi}(t))^{2}+\dot{z}(t)^{2}\right]-\left(\Phi-\frac{r \cdot I_{1}(\kappa \cdot r(t))}{I_{1}(\kappa)} \cdot \Omega \cdot \dot{(t} t\right)\right) \frac{r \cdot I_{1}(\kappa \cdot r(t))}{I_{1}(\kappa)} \cdot \sin (\kappa \cdot z(t)) \\
\Phi=\left(Z \cdot e \cdot \phi_{0}\right) /\left(\frac{m \cdot|\vec{v}|^{2}}{2}\right) \quad \Omega=(Z \cdot e \cdot|\vec{B}| \cdot a) /(m \cdot|\vec{v}|) \\
\kappa=(\pi \cdot a) /\left(2 \cdot L_{\text {Coil }}\right) & \dot{\varphi}(t)=-\Omega \frac{I_{1}(\kappa \cdot r(t))}{\kappa} \cdot \sin (\kappa \cdot z(t))
\end{array}
$$

$\mathrm{I}_{1}$ is the modified first order Bessel-Function. According to Davis et. al. the source is approximated as a point source at the center of the entrance of the solenoid. In the equation all distances are normalized to the radius a of the solenoid, the velocity is normalized to the initial velocity $|\vec{v}|$ and the time is normalized to $a /|\vec{v}|$. Additionally, the Lagrange equation is scaled by a factor $2 / \mathrm{m}$. Because of the low degree of ionization in the IBS process, the charge $\mathrm{Z}$ of the ions is set to one and the mass $\mathrm{m}$ is defined by the atomic mass of the titanium metal and silicon atoms, respectively.

For the description of the curved part, the linear movement in z-direction is replaced by a circular movement and the angular rotation around the angle $\psi$ which is centered in the origin of the bent coil radius. The coordinates are displayed in fig. 2.

The Lagrange equation is modified to:

$$
\begin{gathered}
L=\left[\dot{\rho}(t)^{2}+(\rho(t) \cdot \dot{\psi}(t))^{2}+\dot{\xi}(t)^{2}\right]-\Phi \cdot\left[(\rho(t)-R)^{2}+\xi(t)^{2}\right]+2 \cdot \Omega \cdot(\rho(t)-R) \cdot \dot{\xi}(t) \\
\dot{\varphi}(t)=\omega \cdot r(t)=2 \cdot \pi \cdot f \cdot r(t)
\end{gathered}
$$

Equation (3) can be simplified by using the rotation frequency $\mathrm{f}$ and the angular velocity $\omega$. The definitions of $\Omega$ and $\Phi$ are equivalent to the linear case (Equation (2)).

The equation of motion is determined from the Lagrange equation (2) or (3) by using:

$$
\frac{d}{d t} \frac{\partial L}{\partial \dot{q}_{i}(t)}-\frac{\partial L}{\partial q_{i}(t)}=0
$$

In the linear part the generalized coordinates are given by $\mathrm{q}_{\mathrm{i}}=(\mathrm{r}, \varphi, \mathrm{z})$, and in case of the bent solenoid by $\mathrm{q}_{\mathrm{i}}=(\rho, \psi, \xi)$. Considering typical source characteristics in optical coating technology, the point source can be defined by a Cosinesquare distribution describing the spatial emission, and the distribution of particle energies can be approximated by a Gaussian function. For future simulations the initial conditions have to be replaced and modified to account for specific process conditions. In this context, the point source may be replaced by a spatially extended particle emitter and a Thomson like distribution may be applied to describe the energy of the sputtered ions. In both, the Langrage formalism leads to a system of dependent equations which have to be solved numerically. Fig. 3 displays an example for the calculation using a magnetic field of $6 \mathrm{mT}$ and an electrostatic potential of $40 \mathrm{~V}$. In the plot only the trajectories of the ions, which do not collide with the walls or among each other are displayed. The trajectories of the transmitted ions are assumed to be continuous and differentiable at the interface between the linear and the bent part of the separator. In fig. 3, the trajectories are displayed as a function of the time of flight. Currently, the measurement results are focused on the linear part of the solenoid, and consequently, the theoretical analyses is also addressed to the linear part. 


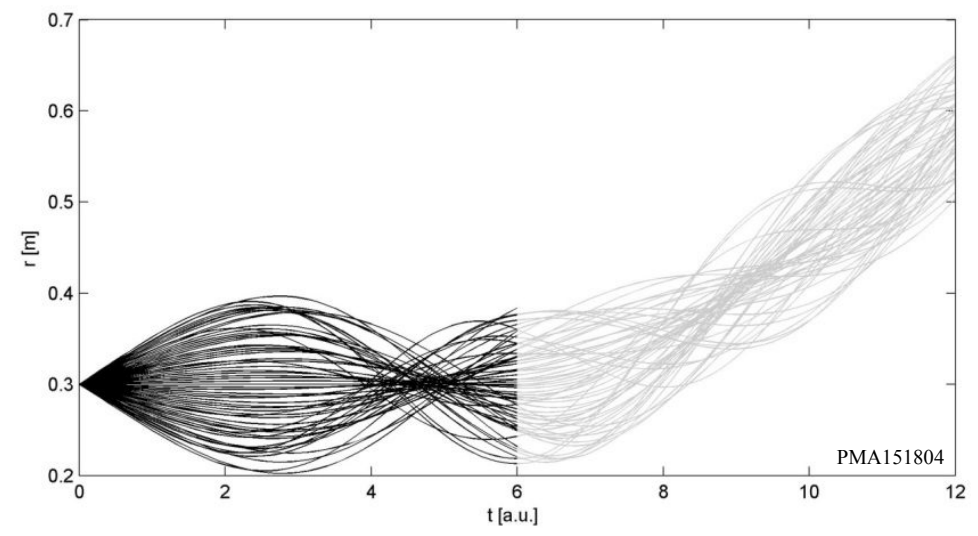

Figure 3: Trajectories inside the separator

The black lines indicate the ion trajectories in the linear transfer coil and the grey lines shows the trajectories in the bent separator coil

\section{EXPERIMENTS AND RESULTS}

To verify the theoretical simulations, experiments have been performed on guiding $\mathrm{TiO}_{2}$ in a transfer coil. In contrast to former studies, where the particle density of the deposited layers was significantly reduced, the major task of the present filtered IBS optimization procedure is an improvement in film quality. Currently an optimization of the layer structure and properties by a controlling the ion properties is targeted. This task requires a detailed understanding of the guiding process. The possibility of a direct measurement of the ion properties is strongly limited, and therefore, the simulation results have to be compared with experimental, macroscopic properties. Especially, guiding efficiencies deliver a possibility for the analyses. The guiding efficiency is determined by deposition experiments on $230 \times 230 \times 1 \mathrm{~mm}^{3}$ borofloat substrates which are firmly mounted at the exit of the transfer coil. As an example, fig. 4 displays the lateral film thickness distribution and the maximum rate of three samples. In this experiment the magnetic field of the transfer coil was varied between -60 and $60 \mathrm{mT}$.

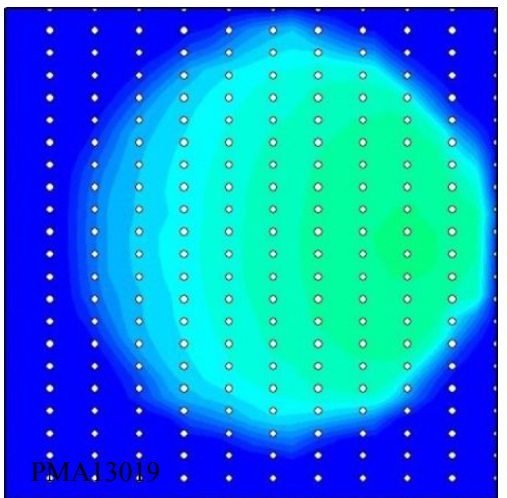

Without magnetic field $\mathrm{r}_{\max }=0.0396 \mathrm{~nm} / \mathrm{s}$

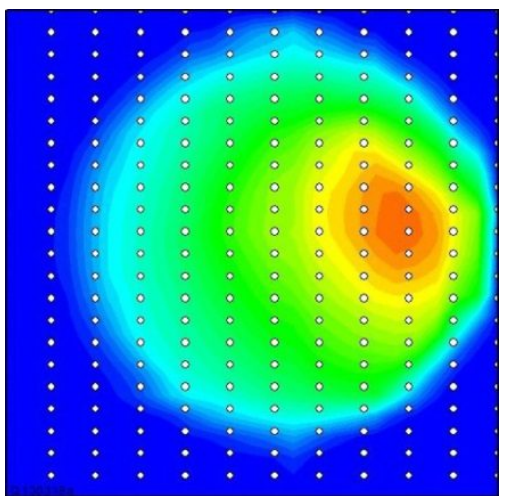

$\mathrm{B}=17,5 \mathrm{mT}$

$\mathrm{r}_{\max }=0.0974 \mathrm{~nm} / \mathrm{s}$
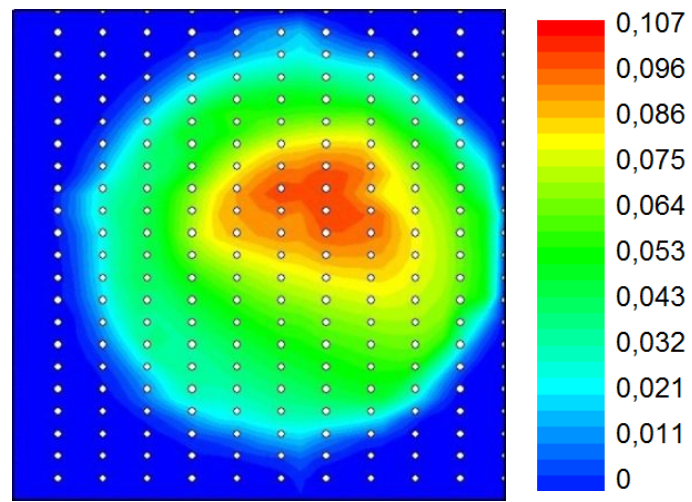

$\mathrm{B}=60 \mathrm{mT}$ $\mathrm{r}_{\max }=0.0999 \mathrm{~nm} / \mathrm{s}$

Figure 4: Lateral film thickness distribution for different magnetic field strengths

The local film thickness is determined by spectral photometric measurements which are evaluated by a conventional thin film algorithm [14]. The measurements indicate that the maximum rate is increasing with higher magnetic field. For the quantitative analyses the average rate $r_{\text {mean }}$ of the deposition process has to be compared to experimental data applying varied field strengths of the separator or varied ion source parameters. The integration of the local rates results in $r_{\text {mean }}$ : 


$$
r_{\text {mean }}=\frac{\int_{0}^{y_{\max }} \int_{0}^{x_{\max }} r(x, y) d x d y}{\int_{0}^{y_{\max }} \int_{0}^{x_{\max }} d x d y}
$$

In fig. 5 the dependency of the normalized maximum rate of a sample on the magnetic field strength is compared to the average rate. In this context, the values are normalized to the maximum value of the measurement series. With respect to the measurement accuracy the measurements match among each other. The rates are starting at the background level without the magnetic field and grow rapidly with increasing magnetic field. In the range between 40 and $50 \mathrm{mT}$ the rates reach their maximum, and saturation can be observed.

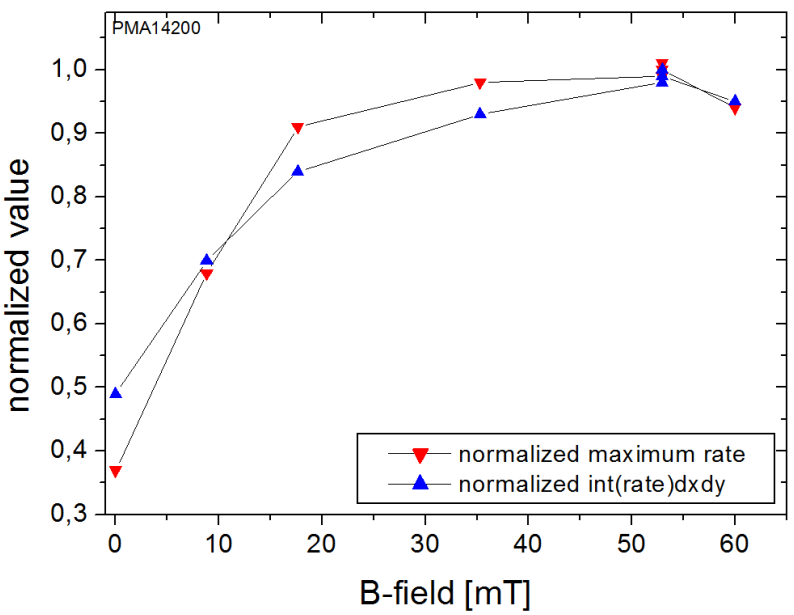

Figure 5: Dependency of the normalized maximum rate and average rate on the magnetic field

In the simulation of the efficiency, the electron-magnetic potential consists of a magnetic part $|\vec{B}|$ and an electronic part $\phi_{0}$. In the Lagrange equations ( 2 and 3 ) both parts are independent. In the experiment the electric potential is influenced by the charge separation which is caused by the magnetic field and consequently, both values are not independent. However, in the simulation the parts are investigated separately. In contrast to the experiments, only ions are considered in the simulation. The guiding efficiency is defined by the ratio between number of ions which enters the coil at the point source and the transient ions at the exit. Fig. 6 displays the calculated trajectories inside the transport coil. In each simulation trajectories of 100 particles are calculated, and the starting conditions are kept constant for the simulation series. Fig. 6 a) illustrates the results without electric and magnetic fields. This calculation corresponds to the transmission of neutral particles. The efficiency is defined by the geometrical masking of the exit aperture. Fig. $6 \mathrm{~b}$ ) and fig. $6 \mathrm{c}$ ) show the behavior for a small electrical field in combination with an electric potential of zero and a strong magnetic field. Thereby, the trajectories of the ions inside the transfer coil are changed only marginal by variation of the magnetic field. In contrast, an increase of the electric potential leads to a significant stronger guiding which can be also observed by the curvatures of the trajectories (fig. $6 \mathrm{~d}$, e and f). 


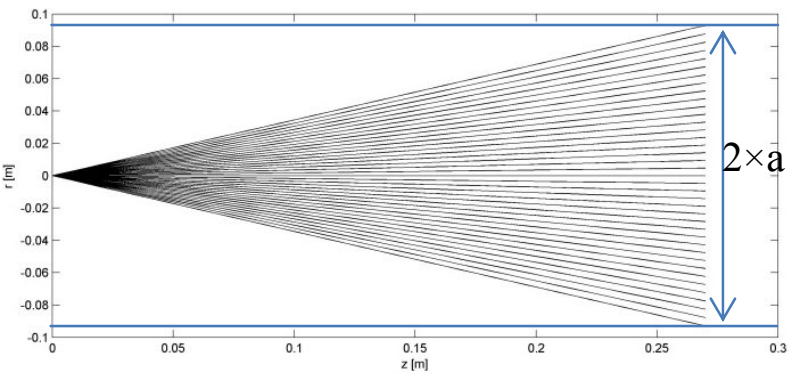

a) $\mathrm{B}=0 \mathrm{mT}$ and $\phi_{0}=0 \mathrm{~V}$

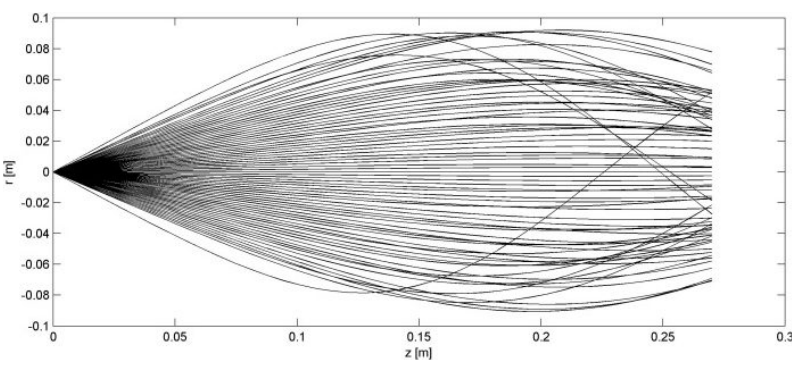

c) $\mathrm{B}=38 \mathrm{mT}$ and $\phi_{0}=7 \mathrm{~V}$

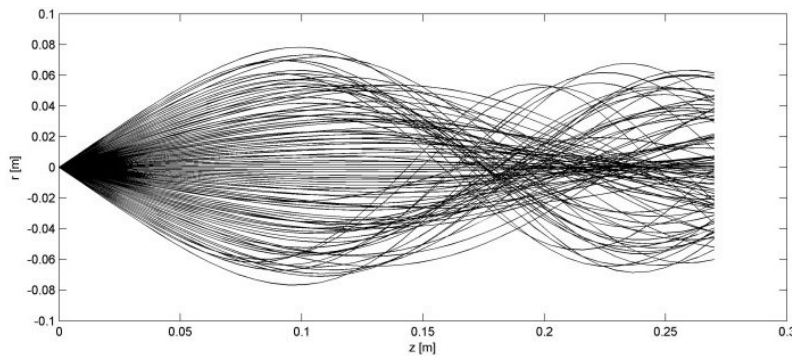

e) $B=57 \mathrm{mT} \phi_{0}=39 \mathrm{~V}$

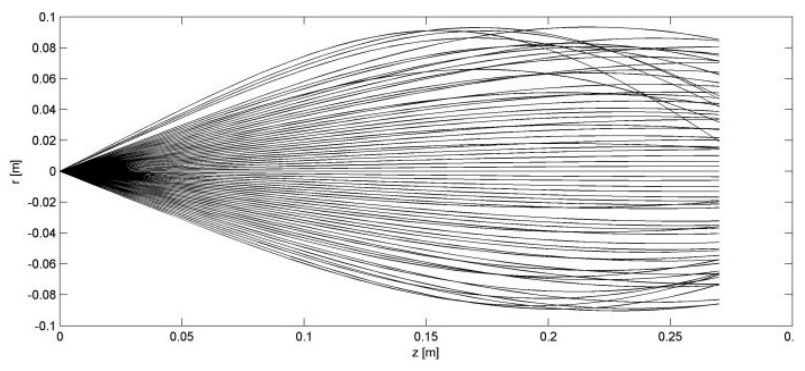

b) $B=0 \mathrm{mT}$ and $\phi_{0}=7 \mathrm{~V}$

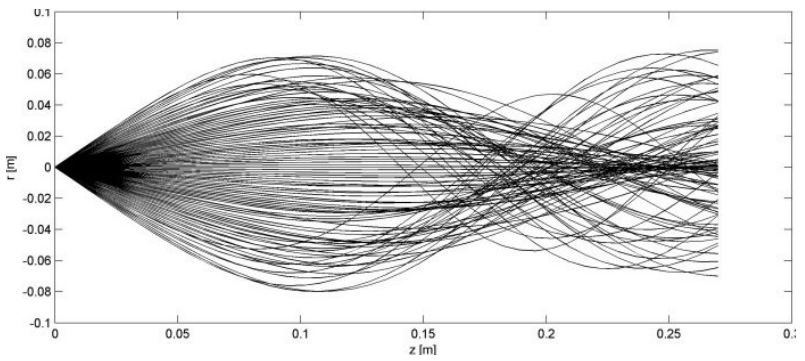

d) $\mathrm{B}=1 \mathrm{mT}$ and $\phi_{0}=39 \mathrm{~V}$

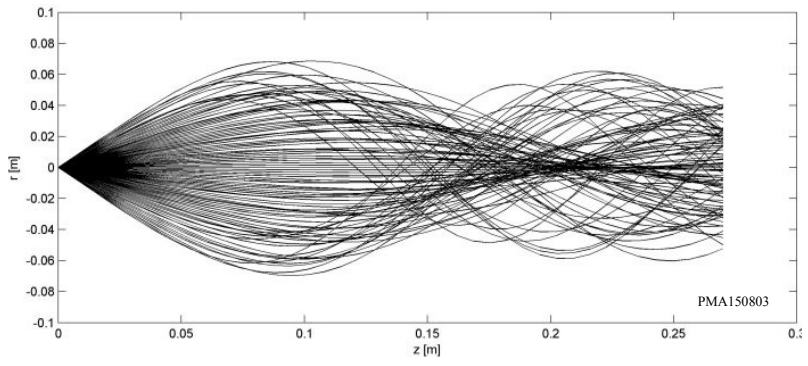

f) $B=65 \mathrm{mT} \phi_{0}=50 \mathrm{~V}$

Figure 6: Calculated trajectories of Titanium ions inside the transfer coil by variation of the external fields

Actually, the ions are only marginally influenced by the magnetic field which is corresponding to the qualitative interpretation according to the Larmor radius of the ions. This behavior necessarily has an impact on the guiding efficiency. The comparison of the experimental curve in fig 5 and the theoretical approximation in fig. 7 indicates that the electrostatic potential determines mainly the properties of the guiding. Especially, the rapid growth in the range of small fields and the significant saturation for high field strengths correlates well to the simulation and the measurements. The dependency of the guiding efficiency on the magnetic field reveals also an increase with the field strength, but the typical rapid increase for low field strength cannot be observed. 


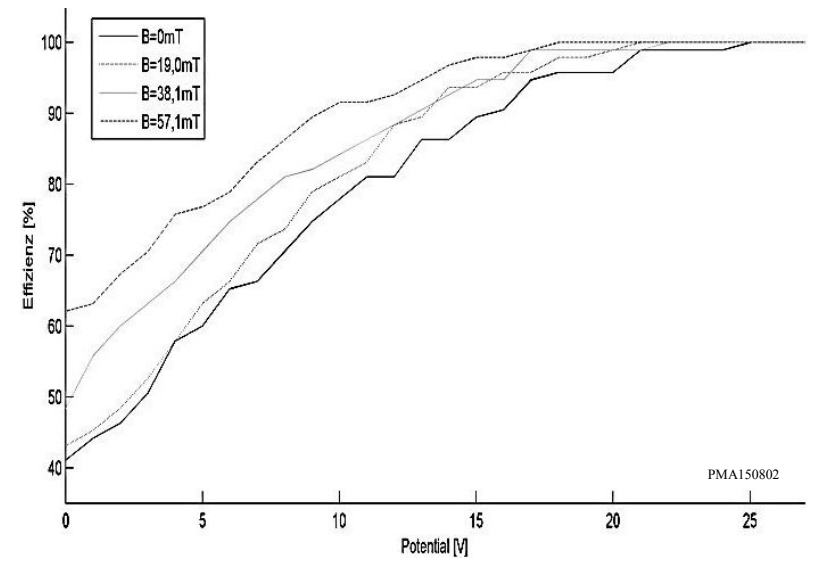

variation of the electric potential

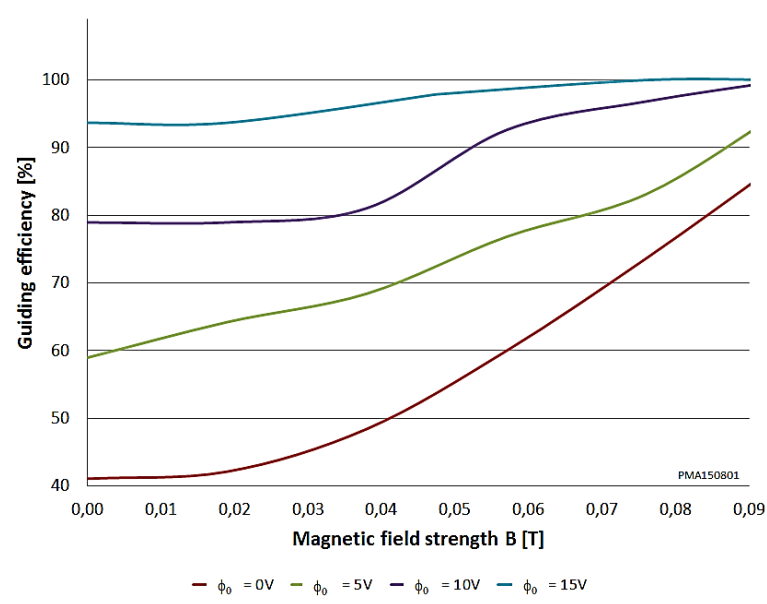

variation of the magnetic field

Figure 7: Theoretical guiding efficiency

The observed guiding efficiency in fig. 7 a) corresponds quantitatively very well to the measured data in fig. 5, which indicates that the approximation in the simulation do not influence the result significantly. Nevertheless, it has to be assumed that the results of different approximations are compensating other effects. For example, the sputtered surface of the target is an expanded area and not a point source. Consequently, the real transition efficiency of the neutrals (without a magnetic field) should be reduced in comparison to the simulation. However, the plasma potential at the wall of the dielectric tube inside the coil can lead to a small guiding effect of the ions, and therefore, the transmission efficiency may be marginally raised. In addition the experimental efficiency decreases after the maximum, marginally. Due to the complex nature these processes are not understood, completely. It can be assumed that the ion-ion interaction or the ionization influences the behavior. In the theoretical description, this behavior cannot be reproduced, but both, the ion-ion interaction as well as the ionization processes are not implemented in the modelling. Nevertheless, the theoretical description of the process is a good basis for a further optimization of the device. Although the simulation overestimates the guiding efficiency, the transfer coils is able to guide the major part of the ions.

\section{SUMMARY}

The present study considers a novel coating process, which spatially separates of the coating material in the sputtering process controlling the thin film growth on the sample surface. In detail, the process applies a magnetic plasma guiding system to transport the coating material from the target to the substrates. The setup includes a bent separator which masks the direct line of sight between the target and the substrates. Generally, only ions of the deposition material are guided by the magnetic field to the substrate. In contrast to this, heavy debris particles follow a linear ballistic trajectory.

The fundamental understanding of the guiding process, the influence of the sputtering and the separator parameters are discussed on the basis of theoretical simulations. In this context, the influence of different parameters of the guiding and of the deposition rate was investigated. The focus of the study was the investigation of influence of the electric potential and the magnetic field on the guiding. On the basis of the simulations, the general assumption was confirmed that the ions are guided mainly by the electrostatic force. In addition, the simulation shows that very high guiding efficiencies can be achieved.

\section{ACKNOWLEDGMENT}

This work was financially supported by the Deutsche Forschungsgemeinschaft (DFG) within the cluster of excellence 201 Quest. 


\section{REFERENCES}

[1] Arenberg, J. W.; Borneis, S.; Cormont, P.; Duparré, A.; Emmert, L. A.; Gruzdev, V.; Hildenbrand-Dhollande, A.; Jupé, M.; Lamaignere, L.and Macleod, A. H.; Mann, K. R.; Mühlig, C.; Néauport, J.; Papernov, S. Riede, W.; Ristau, D.; Rudolph, W.; Schröder, S.; Shao, J.; Shinn, M.; Soileau, M.; Stolz, C. J.; Triebel, W. Wagner, F. R.; Wernham, D. \& Wood, R. M., Ristau, D., ed., "Laser-Induced Damage in Optical Materials," CRC Press (2014).

[2] Kessler, T.; Hagemann, C.; Grebing, C.; Legero, T.; Sterr, U.; Riehle, F.and Martin, M. J.; Chen, L. \& Ye, J., "A sub-40-mHz-linewidth laser based on a silicon single-crystal optical cavity," Nature Photonics 6, 687-692 (2012).

[3] Aksenov, I. I., Belous, V.A., Padalka, V. G., and Khoroshikh, V. M., "Transport of Plasma Streams in a Curvilinear Plasma-Optics System," Soviet. J. Plasma Phys. 4, 425-428, (1978).

[4] Aksenov, I. I., Belokhvostikov, A. N., Padalka, V. G., Repalov, N. S., and Khoroshikh, V.M., " Plasma flux motion in a toroidal plasma guide " Plasma Phys. Controlled Fusion 28, 761-770 (1986).

[5] Bize, S.; Diddams, S.; Tanaka, U.; Tanner, C.; Oskay, W.; Drullinger, R.; Parker, T.; Heavner, T.; Jefferts, S.; Hollberg, L.; Itano; W.M. Wineland, D. \& Bergquist, J., "Testing the stability of fundamental constants with the 199 Hg + single-ion optical clock," Physical Review Letters 90, 1-4 (2003).

[6] Flaminio, R.; Franc, J.; Michel, C.; Morgado, N.; Pinard, L. \& Sassolas, B., "A study of coating mechanical and optical losses in view of reducing mirror thermal noise in gravitational wave detectors," Class. Quantum Grav. 27, 1 to10 (2010).

[7] Anders, A., ed., "Atomic, optical, and plasma physics," Springer Science Business Media (2008).

[8] Johnson, J.; D’Angelo, N. and Merlin, R. L., "Ion-Beam Focusing in a Double-Plasma Device," IEEE Transactions On Plasma Science 16, 590-596 (1988).

[9] Martin, P.; Bendavid, A., "Review of the filtered vacuum arc process and materials deposition," Thin Solid Films 394, 1-15 (2001).

[10] Bohlmark, J.; Östbye, M.; Lattemann, M.; Ljungcrantz, H.; Rosell, T. and U. Helmerson, "Guiding the deposition flux in an ionized magnetron discharge", thin solid films 4, 1928-1931 (2006).

[11] Malobabic, S.; Jupé, M.; Kadkhoda, P. and Ristau, D., "Towards a magnetic field separation in Ion Beam Sputtering processes," Thin Solid Films, 1-5 (2015).

[12] Anders, A.; Anders, S. and Brown, I., "Transport of vacuum arc plasmas through magnetic macroparticle filters," Plasma Sources Sci. Technol. 4, 1-12 (1994).

[13] Davis, C., "Simulation of ion transport through curved-solenoid macroparticle filters," J. Appl. Phys. 72(5), 17401747 (1992).

[14] Dieckmann, M., "Spektrum: Software for optical interference coatings," Laser Zentrum Hannover e.V., www.lzh.de (2002). 